Conclusion At EU level these challenges have been recognised as a priority. ESENER-2 confirms the wide range of $\mathrm{OSH}$ issues identified in other sources and points too at a lower than average use of 'support' services in agriculture, as well as awareness problems. Further analyses of the findings contribute to a better understanding of the challenges and drivers and support policy makers and experts in their future actions to promote a health and safety culture in agriculture.

\section{COHORT STUDY OF MORTALITY AMONG MALE FARM WORKERS IN SPAIN}

${ }^{1} \mathrm{G}$ Zhao*, ${ }^{2} \mathrm{E}$ Regidor, ${ }^{3} \mathrm{E}$ Koselka, ${ }^{1} \mathrm{E}$ Ronda. 'University of Alicante, Alicante, Spain; ${ }^{2}$ Complutense University of Madrid, Madrid, Spain; ${ }^{3}$ Northwestern University, Evanston, USA

\subsection{6/oemed-2018-ICOHabstracts. 1319}

Introduction Jobs in the agricultural sector are associated with high demands, risks, and stress for workers. Spain is of great importance for the European agricultural sector; nearly half the country's land is dedicated to agricultural use. However, the health and mortality risks associated with agricultural work in Spain remain understudied. The aim of this study is to compare the mortality rates for male farm workers with mortality rates for males in non-agricultural occupations in Spain over a ten-year period.

Methods The prospective cohort study followed 8,695,560 male workers in Spain from 2001 to 2010. Participants in the study were selected from the 2001 census; at baseline, all participants were ages 16 to 64 years and employed in an agricultural job. Mortality data included in the present study were obtained from participants' death certificates. For each cause of death, the age-adjusted mortality rates for farm workers and for non-farm workers was calculated per 100000 person-years of follow-up. These rates were used to compare the rate ratio between male farm workers and non-farm workers.

Results The all-cause mortality rate for farm workers was $13 \%$ greater than that of non-farm workers, with a rate radio estimate of 1.13 (95\% CI: 1.11 to 1.16). For cancer deaths, the mortality rates of lip, laryngeal and skin cancer were higher and statistically significant for farm workers. Rates of death resulting from respiratory disease, circulatory disease, accident, and suicide were all significantly higher for farm workers than for non-farm workers.

Discussion These results are the first obtained in Spain from a large prospective agricultural cohort. They show that male farm workers are a vulnerable population in terms of health status. Elevated rates of all-cause and specific-cause mortality in male farm workers could be associated with frequent exposure to occupational hazards, including pesticides, high temperatures, and long working hours.

\section{CIPA-TOX: A NEW TOOL TO ASSESS RETROSPECTIVE OCCUPATIONAL EXPOSURE AND HEALTH EFFECTS TO PESTICIDES IN FRANCE}

\footnotetext{
1,2Johan Spinosi, ${ }^{1,2}$ Anissa Batti, 1,2 Laura Chaperon, ${ }^{1,2}$ Mounia EL Yamani*. 'Direction Santé Travail Agence nationale de santé publique, 12 rue du Val d'Osne 94415 SaintMaurice Cedex France; '2Université Claude Bernard Lyon 1/Umrestt, 8 avenue Rockefeller 69373 Lyon Cedex 08 France
}

10.1136/oemed-2018-ICOHabstracts. 1320
Introduction It is extremely difficult to evaluate the extent to which agricultural workers are exposed to pesticides. This is due to the large number of plant protection products (PPPs) used on a single crop and the variety of active substances (ASs) that have been used over the course of time.

Methods PPP Index is a repertoire of pesticides listing the ASs authorised and marketed in France each year.

All the data collected between 1961 and 2014 was compiled in CIPA-TOX database. Relevant toxicological iformation was added to 1053 ASs. The method used to is based on European regulations, international classification (IARC and US-EPA for carcinogens) and on the search for toxicological reference values (TRV). One or several health effects was attributed to ASs including carcinogenicity, reprotoxicity, neurotoxicity, endocrine disruption, etc.

Results CIPA-Tox provides information about the use of PPP over time in France. The number of ASs authorised, decreased slightly in the second half of the first decade of the millennium; it then remained stable through 2014. For the toxicological field, more than $70 \%$ of the substances authorised in France since 1960 have at least one health effect. Six substances classified as probable or proven carcinogens remain on the market. Endocrine disruptors represent more than 100 authorised ASs. The most affected crops are vinyards and arboriculture.

Discussion The advantages of CIPA-TOX are to take into account all the ASs marketed since 1961 and to apply for identifying health effects a clear and rigorous protocol. The limits are that some substances do not show any health effect. This doesn't mean that they are not harmful but only that the data are lacking. The TRV based on a threshold to avoid the first relevant health effect that appears. This does not mean that other effects do not exist.

\section{IMPROVING EXPOSURE ASSESSMENT METHODOLOGIES FOR EPIDEMIOLOGICAL STUDIES ON PESTICIDES}

${ }^{1}$ Karen Galea, ${ }^{1}$ Ioannis Basinas, ${ }^{1,2}$ John Cherrie, ${ }^{3}$ Roel Vermeulen, ${ }^{3}$ Hans Kromhout, ${ }^{4}$ Kate Jones* ${ }^{4}$ Anne-Helen Harding, ${ }^{5}$ Martie van Tongeren, ${ }^{5}$ Andrew Povey. ${ }^{1}$ Centre for Human Exposure Science (CHES), Institute of Occupational Medicine, Edinburgh, UK; ${ }^{2}$ Heriot Watt University, Institute of Biological Chemistry, Biophysics and Bioengineering, Edinburgh, UK; ${ }^{3}$ Institute for Risk Assessment Sciences, Utrecht University, The Netherlands; ${ }^{4}$ Health and Safety Laboratory, Harpur Hill, Buxton, UK; ${ }^{5}$ Centre for Occupational and Environmental Health, Faculty of Biology, Medicine and Health, University of Manchester, UK

\subsection{6/oemed-2018-ICOHabstracts. 1321}

Introduction The presentation describes a comprehensive study to better understand the reliability of assessment of human exposure to plant protection products (PPP) in previous occupational epidemiological investigations, and to use this information to recommend improvements in scientific practice for future studies. Further, it aims to assess the reliability and external validity of surrogate measures used in these studies to assign exposure to individuals or groups of individuals on the basis of self-reported information, job/crop exposure matrices and exposure algorithms. The project will also evaluate the size and the effects of recall bias on the misclassification of exposure to PPPs and associated health effects.

Methods Existing and newly collected (biological) monitoring data from several existing epidemiological studies and historical records across various populations in Europe, Africa and Asia. Urinary metabolites of pesticides will be selected with due consideration on the extent of use within the study 